\title{
POLYLOGARITHMIC CONNECTIONS WITH EULER SUMS
}

\author{
ANTHONY SOFO
}

\begin{abstract}
Polylogarithmic functions are intrinsically connected with sums of harmonic numbers. In this paper we explore many relations and explicitly derive closed form representations of integrals of polylogarithmic functions and the Lerch phi transcendent in terms of zeta functions and sums of alternating harmonic numbers.
\end{abstract}

\section{INTRODUCTION AND PRELIMINARIES}

In this paper we will develop identities, new families of closed form representations of alternating harmonic numbers and reciprocal binomial coefficients, including integral representations, of the form:

$$
\int_{0}^{1} \frac{x \ln ^{2}(x)}{1-x}{ }_{3} F_{2}\left[\begin{array}{c}
1,1,2 \\
2+k, 2+k
\end{array} \mid-x\right] d x
$$

for $k \geq 1$ and where ${ }_{3} F_{2}\left[\begin{array}{c|c}\cdot, \cdot, \cdot & z \\ \cdot, \cdot & z\end{array}\right]$ is the classical generalized hypergeometric function, also for integrals of the form

$$
\int_{0}^{1} \frac{x \ln ^{2}(x)}{1-x} \Phi(-x, 2,1+r) d x
$$

where $\Phi(z, t, a)=\sum_{m=0}^{\infty} \frac{z^{m}}{(m+a)^{t}}$ is the Lerch transcendent defined for $|z|<1$ and $\mathbb{R}(a)>0$ and satisfies the recurrence

$$
\Phi(z, t, a)=z \Phi(z, t, a+1)+a^{-t} .
$$

2010 Mathematics Subject Classification. Primary: 05A10, 05A19, 33C20; Secondary: 11B65, 11B83, 11M06.

Key words and phrases. Polylogarithm function, integral representation, Lerch transcendent function, alternating harmonic numbers, combinatorial series identities, summation formulas, partial fraction approach, binomial coefficients.

Copyright (c) 2016 by ANUBIH. 
The Lerch transcendent generalizes the Hurwitz zeta function at $z=1$,

$$
\Phi(1, t, a)=\sum_{m=0}^{\infty} \frac{1}{(m+a)^{t}}
$$

and the Polylogarithm, or de Jonquière's function, when $a=1$,

$$
L i_{t}(z):=\sum_{m=1}^{\infty} \frac{z^{m}}{m^{t}}, t \in \mathbb{C} \text { when }|z|<1 ; \mathbb{R}(t)>1 \text { when }|z|=1 .
$$

Moreover

$$
\int_{0}^{1} \frac{L i_{t}(p x)}{x} d x=\left\{\begin{array}{ll}
\zeta(1+t), & \text { for } p=1 \\
\left(2^{-r}-1\right) \zeta(1+t), & \text { for } p=-1
\end{array} .\right.
$$

We also obtain identities for integrals of the type $\int_{0}^{1} \frac{\ln ^{2} x\left(L i_{2}(-x)+x-\frac{x^{2}}{4}\right)}{x^{3}(1-x)} d x$. Let $\mathbb{R}$ and $\mathbb{C}$ denote, respectively the sets of real and complex numbers and let $\mathbb{N}:=\{1,2,3, \ldots\}$ be the set of positive integers, and $\mathbb{N}_{0}:=\mathbb{N} \cup\{0\}$. A generalized binomial coefficient $\left(\begin{array}{l}\lambda \\ \mu\end{array}\right) \quad(\lambda, \mu \in \mathbb{C})$ is defined, in terms of the familiar gamma function, by

$$
\left(\begin{array}{l}
\lambda \\
\mu
\end{array}\right):=\frac{\Gamma(\lambda+1)}{\Gamma(\mu+1) \Gamma(\lambda-\mu+1)}, \quad(\lambda, \mu \in \mathbb{C}),
$$

which, in the special case when $\mu=n, n \in \mathbb{N}_{0}$, yields

$$
\left(\begin{array}{l}
\lambda \\
0
\end{array}\right):=1 \text { and }\left(\begin{array}{l}
\lambda \\
n
\end{array}\right):=\frac{\lambda(\lambda-1) \cdots(\lambda-n+1)}{n !}=\frac{(-1)^{n}(-\lambda)_{n}}{n !}(n \in \mathbb{N}) \text {, }
$$

where $(\lambda)_{\nu}(\lambda, \nu \in \mathbb{C})$ is the Pochhammer symbol. Let

$$
H_{n}=\sum_{r=1}^{n} \frac{1}{r}=\gamma+\psi(n+1),\left(H_{0}:=0\right)
$$

be the $n t h$ harmonic number. Here, as usual, $\gamma$ denotes the Euler-Mascheroni constant and $\psi(z)$ is the Psi (or Digamma) function defined by

$$
\psi(z):=\frac{d}{d z}\{\log \Gamma(z)\}=\frac{\Gamma^{\prime}(z)}{\Gamma(z)} \quad \text { or } \quad \log \Gamma(z)=\int_{1}^{z} \psi(t) d t .
$$

A generalized harmonic number $H_{n}^{(m)}$ of order $m$ is defined, for positive integers $n$ and $m$, as follows:

$$
H_{n}^{(m)}:=\sum_{r=1}^{n} \frac{1}{r^{m}},(m, n \in \mathbb{N}) \quad \text { and } \quad H_{0}^{(m)}:=0 \quad(m \in \mathbb{N})
$$


in terms of integral representations we have the result

$$
H_{n}^{(m+1)}=\frac{(-1)^{m}}{m !} \int_{0}^{1} \frac{(\ln x)^{m}\left(1-x^{n}\right)}{1-x} d x .
$$

In the case of non-integer values of $n$ such as (for example) a value $\rho \in \mathbb{R}$, the generalized harmonic numbers $H_{\rho}^{(m+1)}$ may be defined, in terms of the Polygamma functions

$$
\psi^{(n)}(z):=\frac{d^{n}}{d z^{n}}\{\psi(z)\}=\frac{d^{n+1}}{d z^{n+1}}\{\log \Gamma(z)\} \quad\left(n \in \mathbb{N}_{0}\right),
$$

by

$$
\begin{gathered}
H_{\rho}^{(m+1)}=\zeta(m+1)+\frac{(-1)^{m}}{m !} \psi^{(m)}(\rho+1) \\
(\rho \in \mathbb{R} \backslash\{-1,-2,-3, \ldots\} ; m \in \mathbb{N}),
\end{gathered}
$$

where $\zeta(z)$ is the Riemann zeta function. Whenever we encounter harmonic numbers of the form $H_{\rho}^{(m)}$ at admissible real values of $\rho$, they may be evaluated by means of this known relation (1.4). In the exceptional case of (1.4) when $m=0$, we may define $H_{\rho}^{(1)}$ by

$$
H_{\rho}^{(1)}=H_{\rho}=\gamma+\psi(\rho+1) \quad(\rho \in \mathbb{R} \backslash\{-1,-2,-3, \ldots\}) .
$$

We assume (as above) that

$$
H_{0}^{(m)}=0 \quad(m \in \mathbb{N}) .
$$

In the case of non integer values of the argument $z=\frac{r}{q}$, we may write the generalized harmonic numbers, $H_{z}^{(\alpha+1)}$, in terms of polygamma functions

$$
H_{\frac{r}{q}}^{(\alpha+1)}=\zeta(\alpha+1)+\frac{(-1)^{\alpha}}{\alpha !} \psi^{(\alpha)}\left(\frac{r}{q}+1\right), \frac{r}{q} \neq\{-1,-2,-3, \ldots\},
$$

where $\zeta(z)$ is the zeta function. When we encounter harmonic numbers at possible rational values of the argument, of the form $H_{\frac{r}{q}}^{(\alpha)}$ they maybe evaluated by an available relation in terms of the polygamma function $\psi^{(\alpha)}(z)$ or, for rational arguments $z=\frac{r}{q}$, and we also define

$$
H_{\frac{r}{q}}^{(1)}=\gamma+\psi\left(\frac{r}{q}+1\right) \text {, and } H_{0}^{(\alpha)}=0 .
$$

The evaluation of the polygamma function $\psi^{(\alpha)}\left(\frac{r}{a}\right)$ at rational values of the argument can be explicitly done via a formula as given by Kölbig [9], or Choi and Cvijovic [3] in terms of the Polylogarithmic or other special functions. 
Some specific values are listed in the books[16], [20] and [21]. Let us define the alternating zeta function

$$
\bar{\zeta}(z)=\sum_{n=1}^{\infty} \frac{(-1)^{n+1}}{n^{z}}=\left(1-2^{1-z}\right) \zeta(z)
$$

with $\bar{\zeta}(1)=\ln 2$,

$$
S_{p, q}^{+-}=\sum_{n=1}^{\infty} \frac{(-1)^{n+1} H_{n}^{(p)}}{n^{q}}
$$

For first order powers $(p=1)$, of harmonic numbers, Sitaramachandra Rao [12] gave, for $1+q$ an odd integer,

$$
2 S_{1, q}^{+-}=(1+q) \bar{\zeta}(1+q)-\zeta(1+q)-2 \sum_{j=1}^{\frac{q}{2}-1} \bar{\zeta}(2 j) \zeta(1+q-2 j)
$$

For the positive terms, [1] gave

$$
\begin{aligned}
\sum_{n=1}^{\infty} \frac{H_{n}^{(p)}}{n^{q}} & =\zeta(p) \zeta(q)+\frac{(-1)^{p}}{(p-1) !} \int_{0}^{1} \frac{\ln ^{p-1}(t) L_{i_{q}}(t)}{1-t} d t \\
& =\zeta(p+q)-\frac{(-1)^{q}}{(q-1) !} \int_{0}^{1} \frac{\ln ^{q-1}(t) L_{i_{p}}(t)}{1-t} d t, \text { by symmetry. }
\end{aligned}
$$

Some results for sums of alternating harmonic numbers may be seen in the works of [2], [4], [5], [6], [7], [8], [10], [11], [13], [14], [15], [17], [18], [22], [23], $[24]$ and [25] and references therein.

The following lemma will be useful in the development of the main theorems.

Lemma 1. Let $r$ be a positive integer and $p \in \mathbb{N}$. Then:

$$
\sum_{j=1}^{r} \frac{(-1)^{j}}{j^{p}}=\frac{1}{2^{p}}\left(H_{\left[\frac{r}{2}\right]}^{(p)}+H_{\left[\frac{r-1}{2}\right]}^{(p)}\right)-H_{2\left[\frac{r+1}{2}\right]-1}^{(p)}
$$

where $[x]$ is the integer part of $x$.

Proof. The proof is given in the paper [19].

Lemma 2. The following identities hold. For $0<t \leq 1$

$$
t \ln ^{2}\left(\frac{1+t}{t}\right)=2 \sum_{n=1}^{\infty} \frac{(-t)^{n+1} H_{n}}{n+1}
$$


and when $t=1$,

$$
\begin{gathered}
\ln ^{2} 2=2 \sum_{n=1}^{\infty} \frac{(-1)^{n+1} H_{n}}{n+1}=\zeta(2)-2 L_{i_{2}}\left(\frac{1}{2}\right)=: S_{1} . \\
t \ln \left(\frac{1+t}{t}\right)=\sum_{n=1}^{\infty} \frac{(-t)^{n+1}}{n}, \text { hence } \\
\ln 2=\sum_{n=1}^{\infty} \frac{(-1)^{n+1}}{n}=\sum_{n=1}^{\infty} \frac{1}{n 2^{n}}=\frac{1}{2} \sum_{n=1}^{\infty} \frac{H_{n}}{2^{n}} .
\end{gathered}
$$

Also

$$
\begin{aligned}
& M(0):=\sum_{n=1}^{\infty} \frac{(-1)^{n+1} H_{n}^{(3)}}{n}=\frac{19}{16} \zeta(4)-\frac{3}{4} \ln 2 \zeta(3), \\
& M(1):=\sum_{n=1}^{\infty} \frac{(-1)^{n+1} H_{n}^{(3)}}{n+1}=\frac{3}{4} \ln 2 \zeta(3)-\frac{5}{16} \zeta(4)
\end{aligned}
$$

and

$$
\begin{aligned}
& X(0):=\sum_{n=1}^{\infty} \frac{(-1)^{n+1} H_{n}^{(3)}}{n^{2}}=\frac{3}{4} \zeta(2) \zeta(3)-\frac{21}{32} \zeta(5), \\
& X(1):=\sum_{n=1}^{\infty} \frac{(-1)^{n+1} H_{n}^{(3)}}{(n+1)^{2}}=\frac{51}{32} \zeta(5)-\frac{3}{4} \zeta(2) \zeta(3) .
\end{aligned}
$$

Proof. Firstly (1.6) and (1.7) are standard known results. Next from the definition (1.3),

$$
\begin{aligned}
M(0) & =\sum_{n=1}^{\infty} \frac{(-1)^{n+1} H_{n}^{(3)}}{n}=\frac{1}{2} \int_{0}^{1} \frac{\ln ^{2} x}{1-x} \sum_{n=1}^{\infty} \frac{(-1)^{n+1}\left(1-x^{n}\right)}{n} d x \\
& =\frac{1}{2} \int_{0}^{1} \frac{\ln ^{2} x}{1-x}(\ln 2-\ln (1+x)) d x \\
& =\frac{19}{16} \zeta(4)-\frac{3}{4} \ln 2 \zeta(3) .
\end{aligned}
$$

Here we have used the integral result

$$
\begin{gathered}
\int_{0}^{1} \frac{\ln ^{2} x \ln (1+x)}{1-x} d x=\frac{7}{2} \ln 2 \zeta(3)-\frac{19}{8} \zeta(4) . \\
M(1)=\sum_{n=1}^{\infty} \frac{(-1)^{n+1} H_{n}^{(3)}}{n+1}=\frac{3}{4} \ln 2 \zeta(3)-\frac{5}{16} \zeta(4)
\end{gathered}
$$


follows by a change of counter, also by the integral expression we deduce

$$
\int_{0}^{1} \frac{\ln ^{2} x L i_{2}(-x)}{x(1-x)} d x=\frac{1}{2} \zeta(2) \zeta(3)-\frac{51}{16} \zeta(5)
$$

and hence, (1.8) follows.

In a similar fashion

$$
\begin{aligned}
X(0)= & \sum_{n=1}^{\infty} \frac{(-1)^{n+1} H_{n}^{(3)}}{n^{2}}=\frac{1}{2} \int_{0}^{1} \frac{\ln ^{2} x}{1-x} \sum_{n=1}^{\infty} \frac{(-1)^{n+1}\left(1-x^{n}\right)}{n^{2}} d x \\
= & \frac{1}{2} \int_{0}^{1} \frac{\ln ^{2} x}{1-x}\left(\frac{\zeta(2)}{2}-L i_{2}(-x)\right) d x \\
= & \frac{1}{2} \zeta(2) \zeta(3)-\frac{1}{2} \int_{0}^{1} \frac{\ln ^{2} x L i_{2}(-x)}{1-x} d x=\frac{3}{4} \zeta(2) \zeta(3)-\frac{21}{32} \zeta(5) . \\
& X(1)=\sum_{n=1}^{\infty} \frac{(-1)^{n+1} H_{n}^{(3)}}{(n+1)^{2}}=\frac{51}{32} \zeta(5)-\frac{3}{4} \zeta(2) \zeta(3)
\end{aligned}
$$

follows by a change of counter, or by the integral expression (1.10).

Lemma 3. Let $r \geq 2$ be a positive integer, defining

$$
M(r):=\sum_{n=1}^{\infty} \frac{(-1)^{n+1} H_{n}^{(3)}}{n+r}
$$

then

$$
\begin{aligned}
M(r)=(-1)^{r+1} M(1)+\frac{3(-1)^{r}}{4}\left(H_{r-1}-H_{\left[\frac{r-1}{2}\right]}\right) \zeta(3) \\
\quad+\frac{(-1)^{r+1}}{2}\left(H_{2\left[\frac{r}{2}\right]-1}^{(2)}-\frac{1}{4}\left(H_{\left[\frac{r-1}{2}\right]}^{(2)}+H_{\left[\frac{r-2}{2}\right]}^{(2)}\right)\right) \zeta(2) \\
+(-1)^{r}\left(H_{r-1}^{(3)}+H_{2\left[\frac{r}{2}\right]-1}^{(3)}-\frac{1}{8}\left(H_{\left[\frac{r-1}{2}\right]}^{(3)}+H_{\left[\frac{r-2}{2}\right]}^{(3)}\right)\right) \ln 2 \\
+(-1)^{r+1} \sum_{j=1}^{r-1} \frac{1}{j^{3}}\left(H_{j}-H_{\left[\frac{j}{2}\right]}\right)
\end{aligned}
$$

with $M(0)$ and $M(1)$ given by (1.8). 
Proof. By a change of counter

$$
\begin{aligned}
M(r):= & \sum_{n=1}^{\infty} \frac{(-1)^{n+1} H_{n}^{(3)}}{n+r}=\sum_{n=1}^{\infty} \frac{(-1)^{n} H_{n-1}^{(3)}}{n+r-1} \\
= & \sum_{n=1}^{\infty} \frac{(-1)^{n}}{n+r-1}\left(H_{n}^{(3)}-\frac{1}{n^{3}}\right) \\
= & -\sum_{n=1}^{\infty} \frac{(-1)^{n+1} H_{n}^{(3)}}{n+r-1}+\sum_{n=1}^{\infty} \frac{(-1)^{n+1}}{n^{3}(n+r-1)} \\
= & -M(r-1)+\frac{1}{r-1} \sum_{n=1}^{\infty} \frac{(-1)^{n+1}}{n^{3}}-\frac{1}{(r-1)^{2}} \sum_{n=1}^{\infty} \frac{(-1)^{n+1}}{n^{2}} \\
& +\frac{1}{(r-1)^{3}} \sum_{n=1}^{\infty} \frac{(-1)^{n+1}}{n}-\frac{1}{(r-1)^{3}} \sum_{n=1}^{\infty} \frac{(-1)^{n+1}}{n+r-1} .
\end{aligned}
$$

From Lemma 1 and using the known results,

$$
\begin{aligned}
M(r)= & -M(r-1)+\frac{3 \zeta(3)}{4(r-1)}-\frac{\zeta(2)}{2(r-1)^{2}}+\frac{\ln 2}{(r-1)^{3}} \\
& -\frac{(-1)^{r}}{(r-1)^{3}}\left(\ln 2+H_{r-1}-H_{\left[\frac{r-1}{2}\right]}\right) .
\end{aligned}
$$

From (1.12) we have the recurrence relation

$$
\begin{aligned}
M(r)+M(r-1)=\frac{3 \zeta(3)}{4(r-1)}-\frac{\zeta(2)}{2(r-1)^{2}} & +\left(\frac{1+(-1)^{r}}{(r-1)^{3}}\right) \ln 2 \\
& -\frac{(-1)^{r}}{(r-1)^{3}}\left(H_{\left[\frac{r-1}{2}\right]}-H_{r-1}\right)
\end{aligned}
$$

for $r \geq 2$, and with $M(0)$ and $M(1)$ given by (1.8). The recurrence relation is solved by the subsequent reduction of the

$$
M(r), M(r-1), M(r-2), \ldots, M(1)
$$

terms, finally arriving at the relation (1.11).

Lemma 4. For a positive integer $r \geq 2$, we have the identity

$$
X(r):=\sum_{n=1}^{\infty} \frac{(-1)^{n+1} H_{n}^{(3)}}{(n+r)^{2}}
$$


then

$$
\begin{aligned}
X(r)= & (-1)^{r+1} X(1)+\frac{3(-1)^{r+1}}{4}\left(\frac{1}{4}\left(H_{\left[\frac{r-1}{2}\right]}^{(2)}+H_{\left[\frac{r-2}{2}\right]}^{(2)}\right)-H_{2\left[\frac{r}{2}\right]-1}^{(2)}\right) \zeta \\
& +(-1)^{r}\left(\frac{1}{8}\left(H_{\left[\frac{r-1}{2}\right]}^{(3)}+H_{\left[\frac{r-2}{2}\right]}^{(3)}\right)-H_{2\left[\frac{r}{2}\right]-1}^{(3)}\right) \zeta(2) \\
& +3(-1)^{r}\left(H_{r-1}^{(4)}-\frac{1}{16}\left(H_{\left[\frac{r-1}{2}\right]}^{(4)}+H_{\left[\frac{r-2}{2}\right]}^{(4)}\right)+H_{2\left[\frac{r}{2}\right]-1}^{(4)}\right) \ln 2 \\
& +(-1)^{r} \sum_{j=1}^{r-1}\left(\frac{3}{j^{4}}\left(H_{\left[\frac{j}{2}\right]}-H_{j}\right)+\frac{1}{4 j^{3}}\left(H_{\left[\frac{j}{2}\right]}^{(2)}-H_{\left[\frac{j+1}{2}\right]-\frac{1}{2}}^{(2)}\right)\right),
\end{aligned}
$$

with $X(0)$ and $X(1)$ given by (1.9).

Proof. By expansion, and a change of counter

$$
X(r):=\sum_{n=1}^{\infty} \frac{(-1)^{n+1} H_{n}^{(3)}}{(n+r)^{2}}=\sum_{n=1}^{\infty} \frac{(-1)^{n}\left(H_{n}^{(3)}-\frac{1}{n^{3}}\right)}{(n+r-1)^{2}},
$$

then,

$$
\begin{aligned}
X(r) & =-\sum_{n=1}^{\infty} \frac{(-1)^{n+1} H_{n}^{(3)}}{(n+r-1)^{2}}+\sum_{n=1}^{\infty} \frac{(-1)^{n+1}}{n^{3}(n+r-1)^{2}} \\
& =-X(r-1)+\sum_{n=1}^{\infty}(-1)^{n+1}\left(\begin{array}{c}
\frac{1}{(r-1)^{2} n^{3}}-\frac{2}{(r-1)^{3} n^{2}}+\frac{3}{(r-1)^{4} n} \\
-\frac{1}{(r-1)^{3}(n+r-1)^{2}}-\frac{3}{(r-1)^{4}(n+r-1)}
\end{array}\right) \\
X(r) & =-X(r-1)+\frac{3 \zeta(3)}{4(r-1)^{2}}-\frac{\zeta(2)}{(r-1)^{3}}+\frac{3 \ln 2}{(r-1)^{4}} \\
+ & \frac{(-1)^{r}}{4(r-1)^{3}}\left(H_{\left[\frac{r-1}{2}\right]}^{(2)}-H_{\left[\frac{r}{2}\right]-\frac{1}{2}}^{(2)}\right)+\frac{3(-1)^{r}}{4(r-1)^{4}}\left(\ln 2+H_{\left[\frac{r-1}{2}\right]}-H_{r-1}\right) .
\end{aligned}
$$

Hence we obtain the recurrence relation

$$
\begin{aligned}
& X(r)+X(r-1)=\frac{3 \zeta(3)}{4(r-1)^{2}}-\frac{\zeta(2)}{(r-1)^{3}}+\frac{3\left(1+(-1)^{r}\right) \ln 2}{(r-1)^{4}} \\
& +\frac{3(-1)^{r}}{4(r-1)^{4}}\left(H_{\left[\frac{r-1}{2}\right]}-H_{r-1}\right)+\frac{(-1)^{r}}{4(r-1)^{3}}\left(H_{\left[\frac{r-1}{2}\right]}^{(2)}-H_{\left[\frac{r}{2}\right]-\frac{1}{2}}^{(2)}\right),
\end{aligned}
$$

for $r \geq 2$, and with $X(0)$ and (1) given by (1.9). The recurrence relation (1.14) is solved by the subsequent reduction of the

$$
X(r), X(r-1), X(r-1), \ldots, X(1)
$$


terms, finally arriving at the relation (1.13).

We know develop some integral identities for Lemma 3 and Lemma 4, namely:

Lemma 5. For $r \in \mathbb{N}$,

$$
\begin{aligned}
\int_{0}^{1} \frac{x \ln ^{2}(x)}{1-x}{ }_{2} F_{1}\left[\begin{array}{c}
1,1+r \\
2+r
\end{array} \mid-x\right] d x \\
=(1+r)\left(H_{\frac{r}{2}}-H_{\frac{r-1}{2}}\right) \zeta(3)-2(1+r) M(r)
\end{aligned}
$$

where $M(r)$ is given by (1.11).

Proof. From $M(r):=\sum_{n=1}^{\infty} \frac{(-1)^{n+1} H_{n}^{(3)}}{n+r}$ and by the use of the integral representation (1.3)

$$
\begin{aligned}
M(r) & =\frac{1}{2} \int_{0}^{1} \frac{\ln ^{2}(x)}{1-x} \sum_{n=1}^{\infty} \frac{(-1)^{n+1}\left(1-x^{n}\right)}{n+r} d x \\
& =\frac{1}{2} \int_{0}^{1} \frac{\ln ^{2}(x)}{1-x}\left(\frac{H_{\frac{r}{2}}-H_{\frac{r-1}{2}}}{2}-\frac{x_{2} F_{1}\left[\begin{array}{c}
1,1+r \\
2+r
\end{array} \mid-x\right]}{1+r}\right) d x \\
& =\frac{\zeta(3)}{2}\left(H_{\frac{r}{2}}-H_{\frac{r-1}{2}}\right)-\frac{1}{2(1+r)} \int_{0}^{1} \frac{x \ln ^{2}(x)}{1-x} F_{2}\left[\begin{array}{c}
1,1+r \\
2+r
\end{array} \mid-x\right] d x
\end{aligned}
$$

and by re-arrangement we obtain (1.15).

Remark 1. For the case $r=1$, we have

$$
\int_{0}^{1} \frac{\ln ^{2} x \ln (1+x)}{x(1-x)} d x=\frac{7}{2} \ln 2 \zeta(3)-\frac{5}{8} \zeta(4)
$$

which is a modification of the integral used in Lemma 2. For $r=3$ we obtain the very slow converging integral

$$
\begin{aligned}
\left.\int_{0}^{1} \frac{\ln ^{2} x\left(2 x-x^{2}-2 \ln (1+x)\right)}{x^{3}(1}-x\right) & d x \\
& =\frac{5}{4} \zeta(4)-7 \ln 2 \zeta(3)+\frac{7}{2} \zeta(3)-\frac{3}{2} \zeta(2)+8 \ln 2-\frac{1}{4} .
\end{aligned}
$$

The Wolfram on-line integrator yields no solution to this integral.

Lemma 6. For $r \in \mathbb{N}$,

$$
\int_{0}^{1} \frac{x \ln ^{2}(x)}{1-x} \Phi(-x, 2,1+r) d x=\frac{\zeta(3)}{2}\left(H_{\frac{r}{2}}^{(2)}-H_{\frac{r-1}{2}}^{(2)}\right)-2 X(r)
$$


where $X(r)$ is given by (1.13) and $\Phi(-x, 2,1+r)$ is the Lerch transcendent. Proof. From $X(r):=\sum_{n=1}^{\infty} \frac{(-1)^{n+1} H_{n}^{(3)}}{(n+r)^{2}}$ and by the use of the integral representation $(1.3)$

$$
\begin{aligned}
X(r) & =\frac{1}{2} \int_{0}^{1} \frac{\ln ^{2}(x)}{1-x} \sum_{n=1}^{\infty} \frac{(-1)^{n+1}\left(1-x^{n}\right)}{(n+r)^{2}} d x \\
& =\frac{1}{2} \int_{0}^{1} \frac{\ln ^{2}(x)}{1-x}\left(\frac{H_{\frac{r}{2}}^{(2)}-H_{\frac{r-1}{2}}^{(2)}}{4}-x \Phi(-x, 2,1+r)\right) d x \\
& =\frac{\zeta(3)}{4}\left(H_{\frac{r}{2}}^{(2)}-H_{\frac{r-1}{2}}^{(2)}\right)-\frac{1}{2} \int_{0}^{1} \frac{x \ln ^{2}(x)}{1-x} \Phi(-x, 2,1+r) d x
\end{aligned}
$$

and by re-arrangement we obtain (1.16).

Remark 2. For the case $r=1$, we have

$$
\int_{0}^{1} \frac{\ln ^{2} x L i_{2}(-x)}{x(1-x)} d x=\frac{1}{2} \zeta(2) \zeta(3)-\frac{51}{16} \zeta(5)
$$

which is a modification of the integral used in Lemma 2. The Wolfram on-line integrator yields no solution to this integral.

The next few theorems relate the main results of this investigation, namely the integral and closed form representation of integrals of the type (1.1).

\section{INTEGRAL AND CLOSED FORM IDENTITIES}

In this section we investigate integral identities in terms of closed form representations of infinite series of harmonic numbers of order three and inverse binomial coefficients. First we indicate the closed form representation of

$$
V(k, p, q)=\sum_{n=1}^{\infty} \frac{(-1)^{n+1} H_{n}^{(3)}}{n^{p}\left(\begin{array}{c}
n+k \\
k
\end{array}\right)^{q}}
$$

for six cases $(k, p, q)=(k, 0,1),(k, 1,1),(k, 2,1),(k, 0,2),(k, 1,2),(k, 2,2)$, and $k \geq 1$ is a positive integer.

Theorem 1. Let $k \geq 1$ be real positive integer, then from (2.1) with $p=$ $0,1,2$ and $q=1$ we have:

$$
V(k, 0,1)=\sum_{n=1}^{\infty} \frac{(-1)^{n+1} H_{n}^{(3)}}{\left(\begin{array}{c}
n+k \\
k
\end{array}\right)}=\sum_{r=1}^{k}(-1)^{1+r} r\left(\begin{array}{l}
k \\
r
\end{array}\right) M(r) .
$$




$$
\begin{aligned}
V(k, 1,1)=\sum_{n=1}^{\infty} & \frac{(-1)^{n+1} H_{n}^{(3)}}{n\left(\begin{array}{c}
n+k \\
k
\end{array}\right)} \\
& =\frac{19}{16} \zeta(4)-\frac{3}{4} \ln (2) \zeta(3)-\sum_{r=1}^{k}(-1)^{1+r}\left(\begin{array}{c}
k \\
r
\end{array}\right) M(r),
\end{aligned}
$$

and

$$
\begin{aligned}
V(k, 2,1)=\sum_{n=1}^{\infty} & \frac{(-1)^{n+1} H_{n}^{(3)}}{n^{2}\left(\begin{array}{c}
n+k \\
k
\end{array}\right)}=\frac{3 \zeta(2) \zeta(3)}{4}-\frac{21 \zeta(5)}{32} \\
& -H_{k}\left(\frac{19 \zeta(4)}{16}-\frac{3 \ln 2 \zeta(3)}{4}\right)-\sum_{r=1}^{k} \frac{(-1)^{r}\left(\begin{array}{c}
k \\
r
\end{array}\right) M(r)}{r}
\end{aligned}
$$

where $M(r)$ is given by (1.11).

Proof. Consider the expansion

$$
\begin{aligned}
V(k, 0,1) & =\sum_{n=1}^{\infty} \frac{(-1)^{n+1} H_{n}^{(3)}}{\left(\begin{array}{c}
n+k \\
k
\end{array}\right)}=\sum_{n=1}^{\infty} \frac{(-1)^{n+1} k ! H_{n}^{(3)}}{(n+1)_{k}} \\
& =\sum_{n=1}^{\infty}(-1)^{n+1} k ! H_{n}^{(3)} \sum_{r=1}^{k} \frac{\Theta(r)}{n+r}
\end{aligned}
$$

where

$$
\Theta(r)=\lim _{n \rightarrow-r}\left\{\frac{n+r}{\prod_{r=1}^{k}(n+r)}\right\}=\frac{(-1)^{1+r} r}{k !}\left(\begin{array}{l}
k \\
r
\end{array}\right) .
$$

We can now express

$$
\begin{aligned}
V(k, 0,1) & =\sum_{n=1}^{\infty}(-1)^{n+1} k ! H_{n}^{(3)} \sum_{r=1}^{k} \frac{\Theta(r)}{n+r} \\
& =\sum_{r=1}^{k}(-1)^{1+r} r\left(\begin{array}{l}
k \\
r
\end{array}\right) \sum_{r=1}^{k} \frac{H_{n}^{(3)}}{n+r} .
\end{aligned}
$$

From (1.11) we have $M(r)$, hence substituting into (2.6), (2.2) follows. The identities (2.3) and (2.4) follow in a similar fashion.

The other case of $V(k, p, 2)$, can be evaluated in a similar fashion. We list the result in the next theorem. 
Theorem 2. Under the assumptions of Theorem 1, we have,

$$
\begin{aligned}
& V(k, 0,2)=\sum_{n=1}^{\infty} \frac{(-1)^{n+1} H_{n}^{(3)}}{\left(\begin{array}{c}
n+k \\
k
\end{array}\right)^{2}} \\
&=\sum_{r=1}^{k} r^{2}\left(\begin{array}{c}
k \\
r
\end{array}\right)^{2}\left(M(r)+2\left(H_{r-1}-H_{k-r}\right) X(r)\right), \quad(2.7) \\
& V(k, 1,2)= \sum_{n=1}^{\infty} \frac{(-1)^{n+1} H_{n}^{(3)}}{n\left(\begin{array}{c}
n+k \\
k
\end{array}\right)^{2}} \\
&= \frac{k}{2}\left(\begin{array}{c}
2 k \\
k
\end{array}\right)\left(\frac{19 \zeta(4)}{16}-\frac{3 \ln 2 \zeta(3)}{4}\right)-\sum_{r=1}^{k} r\left(\begin{array}{c}
k \\
r
\end{array}\right)^{2} M(r) \\
&+2 \sum_{r=1}^{k}\left(\begin{array}{c}
k \\
r
\end{array}\right)^{2}\left(H_{r-1}-H_{k-r}\right)\left(\frac{19 \zeta(4)}{16}-\frac{3 \ln 2 \zeta(3)}{4}-M(r)-r X(r)\right)
\end{aligned}
$$

and

$$
\begin{aligned}
V(k, 2,2) & =\sum_{n=1}^{\infty} \frac{(-1)^{n+1} H_{n}^{(3)}}{n^{2}\left(\begin{array}{c}
n+k \\
K
\end{array}\right)^{2}} \\
& =\frac{k}{2}\left(\begin{array}{c}
2 k \\
k
\end{array}\right)\left(\frac{3}{4} \zeta(2) \zeta(3)-\frac{21}{32} \zeta(5)\right) \\
- & \left(\left(\begin{array}{c}
2 k \\
k
\end{array}\right)-1\right)\left(\frac{19 \zeta(4)}{16}-\frac{3 \ln 2 \zeta(3)}{4}\right)+\sum_{r=1}^{k}\left(\begin{array}{c}
k \\
r
\end{array}\right)^{2} M(r) \\
+ & 2 \sum_{r=1}^{k}\left(\begin{array}{c}
k \\
r
\end{array}\right)^{2}\left(H_{r-1}-H_{k-r}\right)\left(\begin{array}{c}
\frac{3}{4} \zeta(2) \zeta(3)-\frac{2}{r}\left(\frac{19 \zeta(4)}{16}-\frac{3 \ln 2 \zeta(3)}{4}\right) \\
-\frac{21}{32} \zeta(5)+\frac{2}{r} M(r)+X(r)
\end{array}\right)
\end{aligned}
$$

where $M(r)$ is given by (1.11) and $X(r)$ is given by (1.13).

Proof. Consider the expansion

$$
\begin{aligned}
V(k, 0,2) & =\sum_{n=1}^{\infty} \frac{(-1)^{n+1} H_{n}^{(3)}}{\left(\begin{array}{c}
n+k \\
k
\end{array}\right)^{2}}=\sum_{n=1}^{\infty} \frac{(-1)^{n+1}(k !)^{2} H_{n}^{(3)}}{\left((n+1)_{1+k}\right)^{2}} \\
& =\sum_{n=1}^{\infty}(-1)^{n+1}(k !)^{2} H_{n}^{(3)} \sum_{r=1}^{k}\left(\frac{\Omega(r)}{n+r}+\frac{\Delta(r)}{(n+r)^{2}}\right)
\end{aligned}
$$


where

$$
\Omega(r)=\lim _{n \rightarrow-r}\left\{\frac{(n+r)^{2}}{\prod_{r=1}^{k}(n+r)^{2}}\right\}=\left(\frac{r}{k !}\left(\begin{array}{l}
k \\
r
\end{array}\right)\right)^{2}
$$

and

$$
\Delta(r)=\lim _{n \rightarrow-r} \frac{d}{d n}\left\{\frac{(n+r)^{2}}{\prod_{r=1}^{k}(n+r)^{2}}\right\}=2\left(\frac{r}{k !}\left(\begin{array}{l}
k \\
r
\end{array}\right)\right)^{2}\left(H_{r-1}-H_{k-r}\right) .
$$

We can now express

$$
V(k, 0,2)=\sum_{n=1}^{\infty}(-1)^{n+1}(k !)^{2} H_{n}^{(3)} \sum_{r=1}^{k}\left(\frac{\Omega(r)}{n+r}+\frac{\Delta(r)}{(n+r)^{2}}\right)
$$

and by substitution (2.7) follows. Similarly (2.8) and (2.9) follow using the same technique.

The following integral identities can be exactly evaluated by using the alternating harmonic number sums in Theorems1 and 2. The following six integral identities are the main results of this paper.

Theorem 3. Let $k$ be a positive integer, then we have:

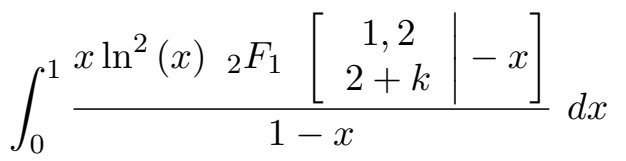

$$
\begin{aligned}
& =2 \zeta(3){ }_{2} F_{1}\left[\begin{array}{c|c}
1,2 & -1 \\
2+k & -1
\end{array}\right]-2(1+k) V(k, 0,1)
\end{aligned}
$$

where $V(k, 0,1)$ is given by $(2.2)$,

$$
\begin{aligned}
& \int_{0}^{1} \frac{x \ln ^{2}(x){ }_{2} F_{1}\left[\begin{array}{c|c}
1,1 \\
2+k
\end{array}\right.}{1-x} d x \\
& =2 \zeta(3){ }_{2} F_{1}\left[\begin{array}{c|c}
1,1 \\
2+k
\end{array} \mid-1\right]-2(1+k) V(k, 1,1),
\end{aligned}
$$

where $V(k, 1,1)$ is given by $(2.3)$,

$$
\begin{aligned}
& \int_{0}^{1} \frac{x \ln ^{2}(x){ }_{3} F_{2}\left[\begin{array}{c}
1,1,1 \\
2,2+k
\end{array}\right.}{1-x} d x \\
& =2 \zeta(3)_{3} F_{2}\left[\begin{array}{c}
1,1,1 \\
2,2+k
\end{array} \mid-1\right]-2(1+k) V(k, 2,1)
\end{aligned}
$$


where $V(k, 2,1)$ is given by $(2.4)$,

$$
\begin{aligned}
& \int_{0}^{1} \frac{x \ln ^{2}(x){ }_{3} F_{2}\left[\begin{array}{c}
1,2,2 \\
2+k, 2+k
\end{array}\right.}{1-x} d x \\
& =2 \zeta(3){ }_{3} F_{2}\left[\begin{array}{c}
1,2,2 \\
2+k, 2+k
\end{array} \mid-1\right]-2(1+k)^{2} V(k, 0,2)
\end{aligned}
$$

where $V(k, 0,2)$ is given by $(2.7)$,

$$
\begin{aligned}
& \int_{0}^{1} \frac{x \ln ^{2}(x){ }_{3} F_{2}\left[\begin{array}{c}
1,1,2 \\
2+k, 2+k
\end{array} \mid-x\right]}{1-x} d x \\
& =2 \zeta(3){ }_{3} F_{2}\left[\begin{array}{c}
1,1,2 \\
2+k, 2+k
\end{array} \mid-1\right]-2(1+k)^{2} V(k, 1,2)
\end{aligned}
$$

where $V(k, 1,2)$ is given by $(2.8)$,

$$
\begin{aligned}
& \int_{0}^{1} \frac{x \ln ^{2}(x){ }_{3} F_{2}\left[\begin{array}{c}
1,1,1 \\
2+k, 2+k
\end{array} \mid-x\right]}{1-x} d x \\
& \quad=2 \zeta(3){ }_{3} F_{2}\left[\begin{array}{c}
1,1,1 \\
2+k, 2+k
\end{array} \mid-1\right]-2(1+k)^{2} V(k, 2,2)
\end{aligned}
$$

where $V(k, 2,2)$ is given by $(2.9)$.

Proof. From the identity (1.3), we can write

$$
\begin{aligned}
& \sum_{n=1}^{\infty} \frac{(-1)^{n+1} H_{n}^{(3)}}{\left(\begin{array}{c}
n+k \\
k
\end{array}\right)}=\frac{1}{2} \int_{0}^{1} \frac{\ln ^{2} x}{1-x} \sum_{n=1}^{\infty} \frac{(-1)^{n+1}\left(1-x^{n}\right)}{\left(\begin{array}{c}
n+k \\
k
\end{array}\right)} d x \\
& =\frac{1}{2(1+k)} \int_{0}^{1} \frac{\ln ^{2} x}{1-x}\left(\begin{array}{c|c}
{ }_{2} F_{1}\left[\begin{array}{c|c}
1,2 & -1 \\
2+k & -1
\end{array}\right] \\
-x_{2} F_{1}\left[\begin{array}{c|c}
1,2 \\
2+k
\end{array}\right.
\end{array}\right) d x .
\end{aligned}
$$

where ${ }_{2} F_{1}[\cdot \cdot \cdot|\cdot| \cdot]$ is the generalized hypergeometric function. Since $\frac{1}{2(1+k)} \int_{0}^{1} \frac{\ln ^{2} x}{1-x}{ }_{2} F_{1}\left[\begin{array}{c}1,2 \\ 2+k\end{array} \mid-1\right] d x=\frac{\zeta(3)}{2(1+k)}{ }_{2} F_{1}\left[\begin{array}{c}1,2 \\ 2+k\end{array} \mid-1\right]$, then by re-arrangement we obtain the integral identity (2.10). The identities (2.11), (2.12), (2.13), (2.14) and (2.15) follow in a similar way. 
Remark 3. From Lemma 6 and Theorem 3 both the Lerch transcendent and the hypergeometric function introduce the polylogarithmic function $L i_{2}(-x)$, from which we are able to evaluate their integrals. The following examples are given. From (1.16) with $r=1$, we obtain (1.17). For $r=2$,

$$
\begin{aligned}
\int_{0}^{1} \frac{\ln ^{2} x\left(L i_{2}(-x)+x\right)}{x^{2}(1-x)} d x=\frac{51}{16} \zeta(5)-\frac{1}{2} \zeta(2) \zeta(3) & -3 \zeta(3) \\
& +\zeta(2)-12 \ln 2+8 .
\end{aligned}
$$

For $r=3$,

$$
\begin{aligned}
\int_{0}^{1} \frac{\ln ^{2} x\left(L i_{2}(-x)+x-\frac{x^{2}}{4}\right)}{x^{3}(1-x)} d x=\frac{51}{16} \zeta(5)- & \frac{1}{2} \zeta(2) \zeta(3)+\frac{3}{8} \zeta(3) \\
& +\frac{5}{8} \zeta(2)-12 \ln 2+\frac{67}{8}
\end{aligned}
$$

and for $r=4$

$$
\begin{aligned}
\int_{0}^{1} \frac{\ln ^{2} x\left(L i_{2}(-x)+x-\frac{x^{2}}{4}+\frac{x^{3}}{9}\right)}{x^{4}(1-x)} d x & =-\frac{51}{16} \zeta(5)+\frac{1}{2} \zeta(2) \zeta(3) \\
+\frac{217}{72} \zeta(3) & -\frac{143}{216} \zeta(2)+\frac{328}{27} \ln 2-\frac{16525}{1944} .
\end{aligned}
$$

The Wolfram on-line integrator, yields no solution to these slow converging integrals.

\section{REFERENCES}

[1] V. Adamchik and H. M. Srivastava, Some series of the zeta and related functions, Analysis, 18 (2) (1998), 131-144.

[2] J. M. Borwein, I. J. Zucker and J. Boersma, The evaluation of character Euler double sums, Ramanujan J., 15 (2008), 377-405.

[3] J. Choi and D. Cvijović, Values of the polygamma functions at rational arguments, J. Phys. A: Math. Theor., 40 (2007), 15019-15028, Corrigendum, ibidem, 43 (2010), 239801 (1 p).

[4] J. Choi, Finite summation formulas involving binomial coefficients, harmonic numbers and generalized harmonic numbers, J. Inequal. Appl., 49 (2013), 11p.

[5] J. Choi and H. M. Srivastava, Some summation formulas involving harmonic numbers and generalized harmonic numbers, Math. Comput. Modelling, 54 (2011), 2220-2234.

[6] M. W. Coffey and N. Lubbers, On generalized harmonic number sums, Appl. Math. Comput., 217 (2010), 689-698.

[7] G. Dattoli and H. M. Srivastava, A note on harmonic numbers, umbral calculus and generating functions, Appl. Math. Lett., 21 (2008), 686-693.

[8] P. Flajolet and B. Salvy, Euler sums and contour integral representations, Exp. Math., 7 (1998), 15-35. 
[9] K. Kölbig, The polygamma function $\psi(x)$ for $x=1 / 4$ and $x=3 / 4$, J. Comput. Appl. Math., 75 (1996), 43-46.

[10] H. Liu and W. Wang, Harmonic number identities via hypergeometric series and Bell polynomials, Integral Transforms Spec. Funct., 23 (2012), 49-68.

[11] I Mezö, Nonlinear Euler sums, Pacific J. Math., 272 (2014), 201-226.

[12] R. Sitaramachandrarao, A formula of S. Ramanujan, J. Number Theory, 25 (1987), $1-19$.

[13] A. Sofo, Sums of derivatives of binomial coefficients, Adv. Appl. Math., 42 (2009), 123-134.

[14] A. Sofo, Integral forms associated with harmonic numbers, Appl. Math. Comput., 207 (2009), 365-372.

[15] A. Sofo, Integral identities for sums, Math. Commun., 13 (2008), 303-309.

[16] A. Sofo, Computational Techniques for the Summation of Series, Kluwer Academic/Plenum Publishers, New York, 2003.

[17] A. Sofo and H. M. Srivastava, Identities for the harmonic numbers and binomial coefficients, Ramanujan J., 25 (2011), 93-113.

[18] A. Sofo, Summation formula involving harmonic numbers, Anal. Math., 379 (2011), 51-64.

[19] A. Sofo, Quadratic alternating harmonic number sums, J. Number Theory, 154 (2015), $144-159$

[20] H. M. Srivastava and J. Choi, Series Associated with the Zeta and Related Functions, Kluwer Academic Publishers, London, 2001.

[21] H. M. Srivastava and J. Choi, Zeta and $q$-Zeta Functions and Associated Series and Integrals, Elsevier Science Publishers, Amsterdam, London and New York, 2012.

[22] W. Wang and C. Jia, Harmonic number identities via the Newton-Andrews method, Ramanujan J., 35 (2014), 263-285.

[23] C. Wei and D. Gong, The derivative operator and harmonic number identities, Ramanujan J., 34 (2014), 361-371.

[24] T. C. Wu, S. T. Tu and H. M. Srivastava, Some combinatorial series identities associated with the digamma function and harmonic numbers, Appl. Math. Lett., 13 (2000), 101-106.

[25] D. Y. Zheng, Further summation formulae related to generalized harmonic numbers, J. Math. Anal. Appl., 335 (1) (2007), 692-706.

(Received: March 22, 2015)

(Revised: June 22, 2015)
Victoria University

P. O. Box 14428

Melbourne City

Victoria 8001

Australia

anthony.sofo@vu.edu.au 